\title{
On the Domain of the Triangle $A(\lambda)$ on the Spaces of Null, Convergent, and Bounded Sequences
}

\author{
Naim L. Braha' ${ }^{1}$ and Feyzi Başar ${ }^{2}$ \\ ${ }^{1}$ Department of Mathematics and Computer Sciences, Avenue "Mother Teresa" Nr=5, 10000 Prishtinë, Kosovo \\ ${ }^{2}$ Department of Mathematics, Faculty of Arts and Sciences, Fatih University, The Hadimköy Campus, \\ Büyükçekmece, 34500 İstanbul, Turkey
}

Correspondence should be addressed to Feyzi Başar; feyzibasar@gmail.com

Received 17 January 2013; Accepted 7 May 2013

Academic Editor: Douglas Anderson

Copyright (c) 2013 N. L. Braha and F. Başar. This is an open access article distributed under the Creative Commons Attribution License, which permits unrestricted use, distribution, and reproduction in any medium, provided the original work is properly cited.

We introduce the spaces of $A(\lambda)$-null, $A(\lambda)$-convergent, and $A(\lambda)$-bounded sequences. We examine some topological properties of the spaces and give some inclusion relations concerning these sequence spaces. Furthermore, we compute $\alpha$-, $\beta$-, and $\gamma$-duals of these spaces. Finally, we characterize some classes of matrix transformations from the spaces of $A(\lambda)$-bounded and $A(\lambda)$-convergent sequences to the spaces of bounded, almost convergent, almost null, and convergent sequences and present a Steinhaus type theorem.

\section{Introduction}

By $\omega$, we denote the space of all complex sequences. If $x \in \omega$, then we simply write $x=\left(x_{k}\right)$ instead of $x=\left(x_{k}\right)_{k=0}^{\infty}$. Also, we will use the conventions that $e=(1,1, \ldots)$, and $e^{(n)}$ is the sequence whose only nonzero term is 1 in the $n$th place for each $n \in \mathbb{N}$, where $\mathbb{N}=\{0,1,2, \ldots\}$. Any vector subspace of $\omega$ is called a sequence space. We will write $\ell_{\infty}, c$ and $c_{0}$ for the sequence spaces of all bounded, convergent, and null sequences, respectively. Further, by $\ell_{p}$ with $1 \leq p<\infty$, we denote the sequence space of all $p$-absolutely convergent series, that is, $\ell_{p}=\left\{x=\left(x_{k}\right) \in \omega: \sum_{k}\left|x_{k}\right|^{p}<\infty\right\}$. For simplicity in notation, here and in what follows, the summation without limits runs from 0 to $\infty$. Moreover, we write $b s$ and $c s$ for the spaces of all bounded and convergent series, respectively. A sequence space $\mu$ is called an FK-space if it is a complete linear metric space with continuous coordinates $p_{n}: \mu \rightarrow \mathbb{C}$, where $\mathbb{C}$ denotes the complex field and $p_{n}(x)=x_{n}$ for all $x=\left(x_{n}\right) \in \mu$ and every $n \in \mathbb{N}$. A normed FK-space is called a BK-space, that is, a BK-space is a Banach space with continuous coordinates. The sequence spaces $c_{0}$ and $c$ are BK-spaces with the usual sup-norm given by $\|x\|_{\infty}=\sup _{n \in \mathbb{N}}\left|x_{n}\right|$. Also, the space $\ell_{p}$ is a BK-space with the usual norm $\|\cdot\|_{p}$ defined by

$$
\|x\|_{p}=\left(\sum_{n}\left|x_{n}\right|^{p}\right)^{1 / p}
$$

where $1 \leq p<\infty$. A sequence $\left(y_{n}\right)$ in a normed space $X$ is called a Schauder basis for $X$ if for every $x \in X$ there is a unique sequence $\left(\alpha_{n}\right)$ of scalars such that $x=\sum_{n} \alpha_{n} y_{n}$, that is,

$$
\lim _{n \rightarrow \infty}\left\|x-\left(\alpha_{0} y_{0}+\alpha_{1} y_{1}+\alpha_{2} y_{2}+\cdots+\alpha_{n} y_{n}\right)\right\|=0 .
$$

The alpha-, beta-, and gamma-duals $\mu^{\alpha}, \mu^{\beta}$, and $\mu^{\gamma}$ of a sequence space $\mu$ are, respectively, defined by

$$
\begin{aligned}
& \mu^{\alpha}=\left\{a=\left(a_{k}\right) \in \omega: a x=\left(a_{k} x_{k}\right) \in \ell_{1} \forall x=\left(x_{k}\right) \in \mu\right\}, \\
& \mu^{\beta}=\left\{a=\left(a_{k}\right) \in \omega: a x=\left(a_{k} x_{k}\right) \in c s \forall x=\left(x_{k}\right) \in \mu\right\}, \\
& \mu^{\gamma}=\left\{a=\left(a_{k}\right) \in \omega: a x=\left(a_{k} x_{k}\right) \in b s \forall x=\left(x_{k}\right) \in \mu\right\} .
\end{aligned}
$$


If $A$ is an infinite matrix with complex entries $a_{n k}$, where $k, n \in \mathbb{N}$, then we write $A=\left(a_{n k}\right)$ instead of $A=\left(a_{n k}\right)_{n, k=0}^{\infty}$. Also, we write $A_{n}$ for the sequence in the $n$th row of the matrix $A$, that is, $A_{n}=\left(a_{n k}\right)_{k=0}^{\infty}$ for every $n \in \mathbb{N}$. Further, if $x=\left(x_{k}\right) \in \omega$ then we define the $A$-transform of $x$ as the sequence $A x=\left\{(A x)_{n}\right\}_{n=0}^{\infty}$, where

$$
(A x)_{n}=\sum_{k} a_{n k} x_{k}
$$

provided the series on the right hand side of (4) convergent for each $n \in \mathbb{N}$.

Furthermore, the sequence $x$ is said to be $A$-summable to $l \in \mathbb{C}$ if $A x$ converges to $l$ which is called the $A$-limit of $x$. In addition, let $\mu$ and $\nu$ be sequence spaces. Then, we say that $A$ defines a matrix mapping from $\mu$ into $\nu$ if for every sequence $x \in \mu$ the $A$-transform of $x$ exists and is in $\nu$. Moreover, we write $(\mu: \nu)$ for the class of all infinite matrices that map $\mu$ into $\nu$. Thus, $A \in(\mu: \nu)$ if and only if $A_{n} \in \mu^{\beta}$ for all $n \in \mathbb{N}$ and $A x \in \nu$ for all $x \in \mu$. The matrix domain $\mu_{A}$ of an infinite matrix $A$ in a sequence space $\mu$ is defined by

$$
\mu_{A}=\{x \in \omega: A x \in \mu\}
$$

which is a sequence space. The approach constructing a new sequence space by means of the matrix domain of a triangle matrix was employed by several authors, see for instance [14]. In this paper, we introduce the spaces of $A(\lambda)$-null, $A(\lambda)$ convergent, and $A(\lambda)$-bounded sequences which generalize the results given in [2]. Further, we define some related BKspaces and construct their bases. Moreover, we establish some inclusion relations concerning those spaces and determine their alpha-, beta-, and gamma-duals. Finally, we characterize some classes of matrix transformations on these sequence spaces.

\section{Notion of $A(\lambda)$-Null, $A(\lambda)$-Convergent, and $A(\lambda)$-Bounded Sequences}

Let $\lambda=\left(\lambda_{k}\right)$ be a strictly increasing sequence of positive real numbers tending to infinity, as $k \rightarrow \infty$ and $\lambda_{n+1} \geq 2 \lambda_{n}$ for each $n \in \mathbb{N}$. From this last relation, it follows that $\Delta^{2} \lambda_{n} \geq 0$. The first and second differences are defined as follows: $\Delta \lambda_{k}=$ $\lambda_{k}-\lambda_{k-1}$ and $\Delta^{2} \lambda_{k}=\Delta\left(\Delta \lambda_{k}\right)=\lambda_{k}-2 \lambda_{k-1}+\lambda_{k-2}$ for all $k \in \mathbb{N}$, where $\lambda_{-1}=\lambda_{-2}=0$.

Let $x=\left(x_{k}\right)$ be a sequence of complex numbers, such that $x_{-1}=x_{-2}=0$. We say that the sequence $x=\left(x_{k}\right)$ is $A(\lambda)$-strongly convergent to a number $l$ if

$$
\lim _{n \rightarrow \infty} \frac{1}{\lambda_{n}-\lambda_{n-1}} \sum_{k=0}^{n}\left|\left(\lambda_{k}-2 \lambda_{k-1}+\lambda_{k-2}\right)\left(x_{k}-l\right)\right|=0 .
$$

This generalizes the concept of $\Lambda$-strong convergence (see [5]).

Lemma 1 (see [5]). A sequence $x=\left(x_{n}\right)$ of complex numbers $\lambda$-strongly converges to a number $l$ if and only if $x=\left(x_{n}\right)$ converges to $l$ in the ordinary sense and

$$
\lim _{n \rightarrow \infty} \frac{1}{\lambda_{n}-\lambda_{n-1}} \sum_{k=0}^{n} \lambda_{k-1}\left|x_{k}-x_{k-1}\right|=0 .
$$

Let us define the sequence $y=\left(y_{n}\right)$ by the $A(\lambda)$ transform of a sequence $x=\left(x_{k}\right)$, that is,

$$
y_{n}=\left(A_{\lambda} x\right)_{n}=\frac{1}{\lambda_{n}-\lambda_{n-1}} \sum_{k=0}^{n}\left(\lambda_{k}-2 \lambda_{k-1}+\lambda_{k-2}\right) x_{k}
$$

for all $n \in \mathbb{N}$. Throughout the text, we suppose that the terms of the sequences $x=\left(x_{k}\right)$ and $y=\left(y_{k}\right)$ are connected with the relation (8).

Lemma 2 (see [5]). If a sequence $\left(y_{n}\right)$ converges to $l$ in the ordinary sense and condition (7) of Lemma 1 holds, then the sequence $x=\left(x_{n}\right)$ of complex numbers $A(\lambda)$-strongly converges to $l$.

Remark 3 (see [5]). From above results, we can conclude the following. The sequence $x=\left(x_{n}\right)$ of complex numbers $A(\lambda)$ strongly converges to $l$ if and only if the following relation holds:

$$
\lim _{n \rightarrow \infty} \frac{1}{\lambda_{n}-\lambda_{n-1}} \sum_{k=0}^{n}\left(\lambda_{k}-2 \lambda_{k-1}+\lambda_{k-2}\right)\left(x_{k}-l\right)=0 .
$$

Now, we define the infinite matrix $A(\lambda)=\left\{a_{n k}(\lambda)\right\}_{n, k=0}^{\infty}$ by

$$
a_{n k}(\lambda)= \begin{cases}\frac{\lambda_{k}-2 \lambda_{k-1}+\lambda_{k-2}}{\lambda_{n}-\lambda_{n-1}}, & 0 \leq k \leq n \\ 0, & k>n\end{cases}
$$

for all $n, k \in \mathbb{N}$. Then, $A(\lambda)$-transform of a sequence $x \in \omega$ is the sequence $A_{\lambda} x=\left\{\left(A_{\lambda} x\right)_{n}\right\}_{n=0}^{\infty}$, where $\left(A_{\lambda} x\right)_{n}$ is given by the relation (8) for every $n \in \mathbb{N}$. Thus, the sequence $x$ is $A(\lambda)$-convergent if and only if $x$ is $A(\lambda)$-summable. Further, if $x$ is $A(\lambda)$-convergent then the $A(\lambda)$-limit of $x$ exists and coincides with the ordinary limit of $x$, that is, to say that the method $A(\lambda)$ is regular.

\section{The Spaces of $A(\lambda)$-Null, $A(\lambda)$-Convergent, and $A(\lambda)$-Bounded Sequences}

We introduce the classes $A_{\lambda}\left(c_{0}\right), A_{\lambda}(c)$, and $A_{\lambda}\left(\ell_{\infty}\right)$ of all $A(\lambda)$-null, $A(\lambda)$-convergent, and $A(\lambda)$-bounded sequences of complex numbers, that is,

$$
\begin{gathered}
A_{\lambda}\left(c_{0}\right)=\left\{x=\left(x_{k}\right) \in \omega: \lim _{n \rightarrow \infty}\left(A_{\lambda} x\right)_{n}=0\right\}, \\
A_{\lambda}(c)=\left\{x=\left(x_{k}\right) \in \omega: \exists l \in \mathbb{C} \ni \lim _{n \rightarrow \infty}\left(A_{\lambda} x\right)_{n}=l\right\}, \\
A_{\lambda}\left(\ell_{\infty}\right)=\left\{x=\left(x_{k}\right) \in \omega: \sup _{n \in \mathbb{N}}\left|\left(A_{\lambda} x\right)_{n}\right|<\infty\right\} .
\end{gathered}
$$

Obviously, $A_{\lambda}\left(c_{0}\right), A_{\lambda}(c)$, and $A_{\lambda}\left(\ell_{\infty}\right)$ are the linear spaces with respect to the usual operations coordinatewise addition and scalar multiplication of sequences. Here and after, by $X$ we denote any of the spaces $c_{0}, c$, and $\ell_{\infty}$. It is not hard to see that the quantity

$$
\|x\|_{A_{\lambda}(X)}:=\sup _{n \in \mathbb{N}} \frac{1}{\lambda_{n}-\lambda_{n-1}} \sum_{k=0}^{n}\left|\left(\lambda_{k}-2 \lambda_{k-1}+\lambda_{k-2}\right) x_{k}\right|
$$


is finite for every $x=\left(x_{k}\right) \in A_{\lambda}(X)$, and $\|\cdot\|_{A_{\lambda}(X)}$ is a norm on $A_{\lambda}(X)$.

Denote by $\|\cdot\|_{b v}$ the usual $b v$-norm, that is, to say that

$$
\|x\|_{b v}:=\sum_{k}\left|x_{k}-x_{k-1}\right| \text {. }
$$

With the notation of (5), we can redefine the spaces $A_{\lambda}\left(c_{0}\right)$, $A_{\lambda}(c)$, and $A_{\lambda}\left(\ell_{\infty}\right)$ as follows:

$$
\begin{gathered}
A_{\lambda}\left(c_{0}\right)=\left(c_{0}\right)_{A(\lambda)}, \\
A_{\lambda}(c)=(c)_{A(\lambda)}, \\
A_{\lambda}\left(\ell_{\infty}\right)=\left(\ell_{\infty}\right)_{A(\lambda)} .
\end{gathered}
$$

Theorem 4. The sequence spaces $A_{\lambda}\left(c_{0}\right), A_{\lambda}(c)$, and $A_{\lambda}\left(\ell_{\infty}\right)$ are $B K$-spaces with the norm given by

$$
\|x\|_{A_{\lambda}(X)}=\left\|A_{\lambda} x\right\|_{\infty}=\sup _{n \in \mathbb{N}}\left|\left(A_{\lambda} x\right)_{n}\right| .
$$

Proof. This follows from Theorem 4.3.12 given in [6] and the relations (14).

Theorem 5. The sequence spaces $A_{\lambda}\left(c_{0}\right), A_{\lambda}(c)$, and $A_{\lambda}\left(\ell_{\infty}\right)$ are norm isomorphic to the spaces $c_{0}, c$, and $\ell_{\infty}$, respectively.

Proof. Since the matrix $A(\lambda)$ is triangle, it has unique inverse which is also triangle matrix (see $[6,1.4 .8]$ ). Therefore, the linear operator, defined by $T: A_{\lambda}(X) \rightarrow X, T x=A_{\lambda} x$ for all $x \in A_{\lambda}(X)$, is bijective and norm preserving by relation (15).

As a consequence of Theorems 4 and 5, we get the following result.

Corollary 6. Define the sequence $e^{(n)}(\lambda) \in A_{\lambda}\left(c_{0}\right)$ for every fixed $n \in \mathbb{N}$ by

$$
e_{k}^{(n)}(\lambda)= \begin{cases}(-1)^{k-n} \frac{\lambda_{n}-\lambda_{n-1}}{\lambda_{k}-2 \lambda_{k-1}+\lambda_{k-2}}, & n \leq k \leq n+1, \\ 0, & \text { otherwise, }\end{cases}
$$

where $k \in \mathbb{N}$. Then, one has the following.

(1) The sequence $\left\{e_{k}^{(0)}(\lambda), e_{k}^{(1)}(\lambda), e_{k}^{(2)}(\lambda), \ldots\right\}$ is a Schauder basis for the space $A_{\lambda}\left(c_{0}\right)$, and every $x \in A_{\lambda}\left(c_{0}\right)$ has a unique representation: $x=\sum_{n}\left(A_{\lambda} x\right)_{n} e_{k}^{(n)}(\lambda)$.

(2) The sequence $\left\{e, e_{k}^{(0)}(\lambda), e_{k}^{(1)}(\lambda), e_{k}^{(2)}(\lambda), \ldots\right\}$ is a Schauder basis for the space $A_{\lambda}(c)$, and every $x \in A_{\lambda}(c)$ has a unique representation: $x=$ le+ $\sum_{n}\left[\left(A_{\lambda} x\right)_{n}-l\right] e_{k}^{(n)}(\lambda)$, where $l=\lim _{n \rightarrow \infty}\left(A_{\lambda} x\right)_{n}$.

\section{Some Inclusion Relations Related to the New Spaces}

In this section, we give some inclusion relations concerning the spaces $A_{\lambda}\left(c_{0}\right), A_{\lambda}(c)$, and $A_{\lambda}\left(\ell_{\infty}\right)$.
Theorem 7. The inclusions $A_{\lambda}\left(c_{0}\right) \subset A_{\lambda}(c) \subset A_{\lambda}\left(\ell_{\infty}\right)$ strictly hold.

Proof. Let us suppose that $x=\left(x_{n}\right) \in A_{\lambda}\left(c_{0}\right)$, then it follows that $x=\left(x_{n}\right) \in A_{\lambda}(c)$ and $x=\left(x_{n}\right) \in A_{\lambda}\left(\ell_{\infty}\right)$. In what follows we show that these inclusions are strict. The first inclusion follows from the fact that every sequence, which converges in ordinary sense, converges in $A(\lambda)$-sense to the same limit. To prove the strictness of the inclusion $A_{\lambda}(c) \subset$ $A_{\lambda}\left(\ell_{\infty}\right)$, define the sequence $x=\left(x_{k}\right)$ by

$$
x_{k}=(-1)^{k} \frac{\lambda_{k}-\lambda_{k-2}}{\lambda_{k}-2 \lambda_{k-1}+\lambda_{k-2}}
$$

for all $k \in \mathbb{N}$. Then, it follows that

$$
\begin{aligned}
\left(A_{\lambda} x\right)_{n} & =\frac{1}{\lambda_{n}-\lambda_{n-1}} \sum_{k=0}^{n}\left(\lambda_{k}-2 \lambda_{k-1}+\lambda_{k-2}\right) x_{k} \\
& =\frac{1}{\lambda_{n}-\lambda_{n-1}} \sum_{k=0}^{n}(-1)^{k}\left(\lambda_{k}-\lambda_{k-2}\right)=(-1)^{n}
\end{aligned}
$$

Therefore, it is trivial that $x=\left(x_{k}\right) \in A_{\lambda}\left(\ell_{\infty}\right) \backslash A_{\lambda}(c)$.

Theorem 8. The equality $A_{\lambda}\left(c_{0}\right) \cap c=c_{0}$ holds.

Proof. First, we prove that $A_{\lambda}\left(c_{0}\right) \cap c \subset c_{0}$. If a sequence $x=\left(x_{n}\right)$ converges in the ordinary sense to $l$ then it follows that $x_{n} \rightarrow l$ converges in $A(\lambda)$-sense, too. This gives the first inclusion. The converse inclusion follows from Lemma 1, in $[5]$.

In what follows we describe some properties of the sequence $\left(\lambda_{n}\right)$ in the space $\ell_{\infty}$.

Theorem 9. For the sequence $\left(\lambda_{n}\right)$ which is given in Section 2, the following relations are satisfied:

(i) $\left(\Delta \lambda_{n-1} /\left(\Delta \lambda_{n}-\Delta \lambda_{n-1}\right)\right)_{n=1}^{\infty} \notin \ell_{\infty}$ if and only if $\liminf _{n \rightarrow \infty}\left(\left(\Delta \lambda_{n+1}-\Delta \lambda_{n}\right) / \Delta \lambda_{n}\right)=0$

(ii) $\left(\Delta \lambda_{n-1} /\left(\Delta \lambda_{n}-\Delta \lambda_{n-1}\right)\right)_{n=1}^{\infty} \in \ell_{\infty}$ if and only if $\liminf _{n \rightarrow \infty}\left(\left(\Delta \lambda_{n+1}-\Delta \lambda_{n}\right) / \Delta \lambda_{n}\right)>0$.

Proof. (i) Let us start with the expression

$$
\begin{aligned}
s_{n}(x) & =x_{n}-\left(A_{\lambda} x\right)_{n} \\
& =\frac{1}{\lambda_{n}-\lambda_{n-1}} \sum_{k=0}^{n}\left(\lambda_{k}-2 \lambda_{k-1}+\lambda_{k-2}\right)\left(x_{n}-x_{k}\right) .
\end{aligned}
$$

After some calculations, we get

$$
\begin{aligned}
s_{n}(x) & =\frac{\Delta \lambda_{n-1}}{\Delta \lambda_{n}}\left[s_{n}(x)+\left(A_{\lambda} x\right)_{n}+\left(A_{\lambda} x\right)_{n-1}\right] \\
& =\frac{\Delta \lambda_{n-1}}{\Delta \lambda_{n}-\Delta \lambda_{n-1}}\left[\left(A_{\lambda} x\right)_{n}-\left(A_{\lambda} x\right)_{n-1}\right] .
\end{aligned}
$$


On the other hand, from the definition of the sequence $\left(\lambda_{n}\right)$ we have

$$
\begin{aligned}
\lambda_{n} \geq 2 \lambda_{n-1} & \Longrightarrow \Delta^{2} \lambda_{n} \geq 0 \\
& \Longrightarrow \Delta \lambda_{n} \geq \Delta \lambda_{n-1} \Longrightarrow \frac{\Delta \lambda_{n}}{\Delta \lambda_{n-1}} \geq 1
\end{aligned}
$$

From the last relation, we have following two possibilities:

(a) $\liminf \operatorname{in\rightarrow \infty }_{n}\left(\left(\Delta \lambda_{n}-\Delta \lambda_{n-1}\right) / \Delta \lambda_{n-1}\right)>0$ or

(b) $\liminf _{n \rightarrow \infty}\left(\left(\Delta \lambda_{n}-\Delta \lambda_{n-1}\right) / \Delta \lambda_{n-1}\right)=0$.

Part (a) is satisfied if and only if $\left(\Delta \lambda_{n-1} /\left(\Delta \lambda_{n}-\right.\right.$ $\left.\left.\Delta \lambda_{n-1}\right)\right)_{n=1}^{\infty}$ is bounded. Part (b) is satisfied if and only if $\left(\Delta \lambda_{n-1} /\left(\Delta \lambda_{n}-\Delta \lambda_{n-1}\right)\right)_{n=1}^{\infty}$ is unbounded.

Lemma 10. The inclusions $c_{0} \subset A_{\lambda}\left(c_{0}\right)$ and $c \subset A_{\lambda}(c)$ hold. Those spaces coincide if and only if $s(x) \in c_{0}$ for every $x \in$ $A_{\lambda}\left(c_{0}\right)$, respectively, $A_{\lambda}(c)$, where $s(x)=\left\{s_{n}(x)\right\}_{n=0}^{\infty}$.

Lemma 11. The inclusion $\ell_{\infty} \subset A_{\lambda}\left(\ell_{\infty}\right)$ holds. Those spaces coincide if and only if $s(x) \in \ell_{\infty}$ for every $x \in A_{\lambda}\left(\ell_{\infty}\right)$.

Theorem 12. The inclusions $c_{0} \subset A_{\lambda}\left(c_{0}\right), c \subset A_{\lambda}(c)$ and $\ell_{\infty} \subset$ $A_{\lambda}\left(\ell_{\infty}\right)$ strictly hold if and only if

$$
\liminf _{n \rightarrow \infty} \frac{\Delta \lambda_{n+1}-\Delta \lambda_{n}}{\Delta \lambda_{n}}>0
$$

Proof. Let us suppose that $\ell_{\infty} \subset A_{\lambda}\left(\ell_{\infty}\right)$ is strict. Then, from Lemma 11, it follows that there exists a sequence $x=\left(x_{n}\right) \in$ $A_{\lambda}\left(\ell_{\infty}\right)$ such that $s(x)=\left\{s_{n}(x)\right\}_{n=0}^{\infty} \notin \ell_{\infty}$. Since $x=\left(x_{n}\right) \in$ $A_{\lambda}\left(\ell_{\infty}\right)$, we have $A_{\lambda} x \in \ell_{\infty}$ which leads us to the fact that $\left\{x_{n}-s_{n}(x)\right\} \in \ell_{\infty}$. On the other hand, from relation (20), it follows that $\left(\Delta \lambda_{n-1} /\left(\Delta \lambda_{n}-\Delta \lambda_{n-1}\right)\right) \notin \ell_{\infty}$. The last relation is equivalent to

$$
\liminf _{n \rightarrow \infty} \frac{\Delta \lambda_{n+1}-\Delta \lambda_{n}}{\Delta \lambda_{n}}=0
$$

by part (i) of Theorem 9. In a similar way we can conclude that the inclusions $c_{0} \subset A_{\lambda}\left(c_{0}\right), c \subset A_{\lambda}(c)$ are strict. In what follows we prove the sufficiency. Let

$$
\liminf _{n \rightarrow \infty} \frac{\Delta \lambda_{n+1}-\Delta \lambda_{n}}{\Delta \lambda_{n}}=0
$$

Then, from, part (i) of Theorem 9, it follows that $\left(\Delta \lambda_{n-1} /\right.$ $\left.\left(\Delta \lambda_{n}-\Delta \lambda_{n-1}\right)\right) \notin \ell_{\infty}$ and $\left(\left(\Delta \lambda_{n-1}+\Delta \lambda_{n}\right) /\left(\Delta \lambda_{n}-\Delta \lambda_{n-1}\right)\right) \notin \ell_{\infty}$. Let us define the sequence $x=\left(x_{n}\right)$ by

$$
x_{n}=(-1)^{n} \frac{\Delta \lambda_{n-1}+\Delta \lambda_{n}}{\Delta \lambda_{n}-\Delta \lambda_{n-1}}
$$

for all $n \in \mathbb{N}$. Then, we get the following estimation:

$$
\begin{aligned}
\left|\left(A_{\lambda} x\right)_{n}\right|=\frac{1}{\lambda_{n}-\lambda_{n-1}} \mid \sum_{k=0}^{n}(-1)^{k} \frac{\Delta \lambda_{k-1}+\Delta \lambda_{k}}{\Delta \lambda_{k}-\Delta \lambda_{k-1}} & \\
& \times\left(\lambda_{k}-2 \lambda_{k-1}+\lambda_{k-2}\right) \mid \\
= & \frac{1}{\lambda_{n}-\lambda_{n-1}}\left|\sum_{k=0}^{n}(-1)^{k}\left(\Delta \lambda_{k-1}+\Delta \lambda_{k}\right)\right| \\
= & \frac{1}{\lambda_{n}-\lambda_{n-1}}\left|(-1)^{n} \Delta \lambda_{n}\right|=1 .
\end{aligned}
$$

Hence, $A_{\lambda} x \in \ell_{\infty}$ which means that $x \in A_{\lambda}\left(\ell_{\infty}\right) \backslash \ell_{\infty}$. If $\liminf _{n \rightarrow \infty}\left(\left(\Delta \lambda_{n+1}-\Delta \lambda_{n}\right) / \Delta \lambda_{n}\right)=0$. Then, there exists a subsequence $\left(n_{r}\right)$ such that

$$
\lim _{r \rightarrow \infty} \frac{\Delta \lambda_{n_{r}+1}-\Delta \lambda_{n_{r}}}{\Delta \lambda_{n_{r}}}=0 .
$$

Now, let us define the sequence $x=\left(x_{n}\right)$ by

$$
x_{n}= \begin{cases}1, & n=n_{r} \\ -\frac{\Delta \lambda_{k-1}-\Delta \lambda_{k-2}}{\Delta \lambda_{k}-\Delta \lambda_{k-1}}, & n=n_{r} \\ 0, & \text { otherwise }\end{cases}
$$

for all $n \in \mathbb{N}$. It follows from (28) that $x \notin c$. On the other hand,

$$
\left(A_{\lambda} x\right)_{n}= \begin{cases}\frac{\Delta \lambda_{k}-\Delta \lambda_{k-1}}{\Delta \lambda_{k}}, & n=n_{r} \\ 0, & \text { otherwise. }\end{cases}
$$

Now, from the relations (27) and (29), we derive that $x=$ $\left(x_{n}\right) \in A_{\lambda}\left(c_{0}\right) \subset A_{\lambda}(c)$. This completes the proof.

As an immediate result of Theorem 12, we have the following.

Corollary 13. The equalities $c_{0}=A_{\lambda}\left(c_{0}\right), c=A_{\lambda}(c)$, and $\ell_{\infty}=A_{\lambda}\left(\ell_{\infty}\right)$ are satisfied if and only if

$$
\liminf _{n \rightarrow \infty} \frac{\Delta \lambda_{n+1}-\Delta \lambda_{n}}{\Delta \lambda_{n}}>0
$$

Proposition 14. The following statements hold.

(i) Although $c$ and $A_{\lambda}\left(c_{0}\right)$ overlap, the space $A_{\lambda}\left(c_{0}\right)$ does not include the space $c$.

(ii) Although $\ell_{\infty}$ and $A_{\lambda}(c)$ overlap, the space $A_{\lambda}(c)$ does not include the space $\ell_{\mathrm{o}}$.

Proposition 15. If $\liminf _{n \rightarrow \infty}\left(\left(\Delta \lambda_{n+1}-\Delta \lambda_{n}\right) / \Delta \lambda_{n}\right)=0$, then the following statements hold.

(i) Neither of the spaces $c$ and $A_{\lambda}\left(c_{0}\right)$ includes the other.

(ii) Neither of the spaces $A_{\lambda}\left(c_{0}\right)$ and $\ell_{\infty}$ includes the other.

(iii) Neither of the spaces $A_{\lambda}(c)$ and $\ell_{\infty}$ includes the other. 


\section{The $\alpha$-, $\beta$-, and $\gamma$-Duals of the Spaces $A_{\lambda}\left(c_{0}\right)$, $A_{\lambda}(c)$, and $A_{\lambda}\left(\ell_{\infty}\right)$}

In this section, we determine the alpha-, beta-, and gammaduals of the spaces $A_{\lambda}\left(c_{0}\right), A_{\lambda}(c)$, and $A_{\lambda}\left(\ell_{\infty}\right)$.

We need the following lemma due to Stieglitz and Tietz [3] in proving Theorem 17.

Lemma 16. $A=\left(a_{n k}\right) \in\left(c_{0}: \ell_{1}\right)=\left(c: \ell_{1}\right)$ if and only if

$$
\sup _{K, N \in \mathscr{F}}\left|\sum_{n \in N} \sum_{k \in K} a_{n k}\right|<\infty
$$

Here and after, by $\mathscr{F}$ one denotes the collection of all finite subsets of $\mathbb{N}$.

Theorem 17. The $\alpha$-dual of the spaces $A_{\lambda}\left(c_{0}\right), A_{\lambda}(c)$, and $A_{\lambda}\left(\ell_{\infty}\right)$ is the set

$$
a_{1}(\lambda)=\left\{a=\left(a_{n}\right) \in \omega: \sum_{n} \frac{\lambda_{n}-\lambda_{n-1}}{\lambda_{n}-2 \lambda_{n-1}+\lambda_{n-2}}\left|a_{n}\right|<\infty\right\} .
$$

Proof. Define the matrix $B=\left(b_{n k}\right)$ with the aid of a sequence $a=\left(a_{n}\right)$ as follows:

$$
\begin{aligned}
& b_{n k} \\
& = \begin{cases}(-1)^{n-k} \frac{\lambda_{k}-\lambda_{k-1}}{\lambda_{n}-2 \lambda_{n-1}+\lambda_{n-2}} a_{n}, & n-1 \leq k \leq n, \\
0, & 0 \leq k \leq n-1 \text { or } k>n .\end{cases}
\end{aligned}
$$

Then, $x=\left(x_{n}\right) \in A_{\lambda}\left(c_{0}\right)$, we have from Theorem 5

$$
a_{n} x_{n}=a_{n} \sum_{k=n-1}^{n}(-1)^{n-k} \frac{\lambda_{k}-\lambda_{k-1}}{\lambda_{n}-2 \lambda_{n-1}+\lambda_{n-2}} y_{k}=(B y)_{n}
$$

for all $n \in \mathbb{N}$. From the relation (34), it follows that $a x=$ $\left(a_{n} x_{n}\right) \in \ell_{1}$ whenever $x \in A_{\lambda}\left(c_{0}\right)$ if and only if $B y \in \ell_{1}$ whenever $y=\left(y_{k}\right) \in c_{0}$, that is, $a \in\left\{A_{\lambda}\left(c_{0}\right)\right\}^{\alpha}$ if and only if $B \in\left(c_{0}: \ell_{1}\right)$. By Lemma 16 , this is possible if and only if

$$
\sup _{K, N \in \mathscr{F}}\left|\sum_{n \in N} \sum_{k \in K} b_{n k}\right|<\infty .
$$

Now, from definition of the sets $K, N$ and the matrix $B=$ $\left(b_{n k}\right)$, it follows that (35) holds if and only if

$$
\sum_{n} \frac{\lambda_{n}-\lambda_{n-1}}{\lambda_{n}-2 \lambda_{n-1}+\lambda_{n-2}}\left|a_{n}\right|<\infty
$$

which gives that $\left\{A_{\lambda}\left(c_{0}\right)\right\}^{\alpha}=a_{1}(\lambda)$.

In a similar way, one can show that $a_{1}(\lambda)$ is the $\alpha$-dual of the spaces $A_{\lambda}(c)$ and $A_{\lambda}\left(\ell_{\infty}\right)$. So, we omit the details.
Theorem 18. Define the sets $A, B, C$, and $D$ as follows:

$$
\begin{gathered}
A=\left\{a=\left(a_{k}\right) \in \omega:\right. \\
\left.\quad \sum_{k}\left|\Delta\left[\frac{a_{k}}{\lambda_{k}-2 \lambda_{k-1}+\lambda_{k-2}}\left(\lambda_{k}-\lambda_{k-1}\right)\right]\right|\right\}, \\
B=\left\{a=\left(a_{k}\right) \in \omega: \sup _{k \in \mathbb{N}}\left|\frac{a_{k}\left(\lambda_{k}-\lambda_{k-1}\right)}{\lambda_{k}-2 \lambda_{k-1}+\lambda_{k-2}}\right|\right\}, \\
C=\left\{a=\left(a_{k}\right) \in \omega: \lim _{k \rightarrow \infty} \frac{a_{k}\left(\lambda_{k}-\lambda_{k-1}\right)}{\lambda_{k}-2 \lambda_{k-1}+\lambda_{k-2}} \text { exists }\right\}, \\
D=\left\{a=\left(a_{k}\right) \in \omega: \lim _{k \rightarrow \infty} \frac{a_{k}\left(\lambda_{k}-\lambda_{k-1}\right)}{\lambda_{k}-2 \lambda_{k-1}+\lambda_{k-2}}=0\right\} .
\end{gathered}
$$

Then, one has $\left\{A_{\lambda}\left(c_{0}\right)\right\}^{\beta}=A \cap B,\left\{A_{\lambda}(c)\right\}^{\beta}=A \cap C$ and $\left\{A_{\lambda}\left(\ell_{\infty}\right)\right\}^{\beta}=A \cap D$.

Proof. Since the proof is similar for the spaces $A_{\lambda}\left(c_{0}\right)$ and $A_{\lambda}\left(\ell_{\infty}\right)$, we consider only the space $A_{\lambda}(c)$. Let $u=\left(u_{k}\right) \in$ $\omega$. Then, taking into account the relation (8) between the sequences $x=\left(x_{k}\right)$ and $y=\left(y_{k}\right)$, we obtain that

$$
\begin{aligned}
\sum_{k=0}^{n} u_{k} x_{k}= & \sum_{k=0}^{n} u_{k} \sum_{j=k-1}^{k}(-1)^{k-j} \frac{\lambda_{j}-\lambda_{j-1}}{\lambda_{k}-2 \lambda_{k-1}+\lambda_{k-2}} y_{j} \\
= & \sum_{k=0}^{n-1}\left(\lambda_{k}-\lambda_{k-1}\right) \Delta\left(\frac{u_{k}}{\lambda_{k}-2 \lambda_{k-1}+\lambda_{k-2}}\right) y_{k} \\
& +\frac{\left(\lambda_{n}-\lambda_{n-1}\right) u_{n} y_{n}}{\lambda_{n}-2 \lambda_{n-1}+\lambda_{n-2}} \\
= & (B y)_{n}, \quad \forall n \in \mathbb{N},
\end{aligned}
$$

where

$$
\begin{aligned}
\Delta\left(\frac{u_{k}}{\lambda_{k}-2 \lambda_{k-1}+\lambda_{k-2}}\right)= & \frac{u_{k}}{\lambda_{k}-2 \lambda_{k-1}+\lambda_{k-2}} \\
& -\frac{u_{k+1}}{\lambda_{k+1}-2 \lambda_{k}+\lambda_{k-1}},
\end{aligned}
$$

and the matrix $B=\left(b_{n k}\right)$ is defined by

$$
b_{n k}= \begin{cases}\left(\lambda_{k}-\lambda_{k-1}\right) & \\ \times \Delta\left(\frac{u_{k}}{\lambda_{k}-2 \lambda_{k-1}+\lambda_{k-2}}\right), & 0 \leq k \leq n-1, \\ \frac{\left(\lambda_{n}-\lambda_{n-1}\right) u_{n}}{\lambda_{n}-2 \lambda_{n-1}+\lambda_{n-2}}, & k=n, \\ 0, & k>n\end{cases}
$$

for all $k, n \in \mathbb{N}$. Therefore, one can easily see from (38) that $u x=\left(u_{k} x_{k}\right) \in c s$ with $x=\left(x_{k}\right) \in A_{\lambda}(c)$ if and only if $B y \in$ $c$ with $y=\left(y_{k}\right) \in c$, where $B=\left(b_{n k}\right)$ is defined by (40). That is, to say that $u=\left(u_{k}\right) \in\left\{A_{\lambda}(c)\right\}^{\beta}$ if and only if $B$ is 
a matrix satisfying the conditions of Kojima-Schur's theorem (cf. Başar [7, Theorem 3.3.3, page 35]). This leads to the fact that $\left\{A_{\lambda}(c)\right\}^{\beta}=A \cap C$.

Theorem 19. The $\gamma$-dual of the spaces $A_{\lambda}\left(c_{0}\right), A_{\lambda}(c)$, and $A_{\lambda}\left(\ell_{\infty}\right)$ is the set $A \cap B$.

Proof. This is similar to the proof of Theorem 18. So, we omit the details.

\section{Some Matrix Transformation Related to \\ Sequence Spaces $A_{\lambda}\left(c_{0}\right), A_{\lambda}(c)$, and $A_{\lambda}\left(\ell_{\infty}\right)$}

In this section, we characterize the matrix transformations from the spaces $A_{\lambda}\left(\ell_{\infty}\right)$ and $A_{\lambda}(c)$ into the spaces $\ell_{\infty}$, $f, f_{0}, c$, and $c_{0}$ of bounded, almost convergent, almost null, convergent, and null sequences, respectively. We write throughout for brevity that

$$
\begin{gathered}
\Delta a_{n k}=a_{n k}-a_{n, k+1}, \\
\tilde{a}_{n k}=\left(\lambda_{k}-\lambda_{k-1}\right) \Delta\left(\frac{a_{n k}}{\lambda_{k}-2 \lambda_{k-1}+\lambda_{k-2}}\right), \\
a(n, k)=\sum_{j=0}^{n} a_{j k}, \\
c_{n k}=\sum_{j=0}^{n} \frac{1}{j+1} a_{j k}, \\
d_{n k}=s a_{n-1, k}+r a_{n k}, \\
e_{n k}=t a_{n-2, k}+s a_{n-1, k}+r a_{n k}, \\
a(n, k, m)=\frac{1}{m+1} \sum_{j=0}^{m} a_{n+j, k}
\end{gathered}
$$

for all $k, m, n \in \mathbb{N}$, and we use these abbreviations with other letters, where $r, s, t \in \mathbb{R} \backslash\{0\}$.

Theorem 20. $A=\left(a_{n k}\right) \in\left(A_{\lambda}(X): \ell_{\infty}\right)$ if and only if

$$
\begin{gathered}
\left(\frac{\lambda_{k}-\lambda_{k-1}}{\lambda_{k}-2 \lambda_{k-1}+\lambda_{k-2}} a_{n k}\right)_{k \in \mathbb{N}} \in c_{0} \quad \text { for each fixed } n \in \mathbb{N} \\
\sup _{n \in \mathbb{N}} \sum_{k}\left|\tilde{a}_{n k}\right|<\infty .
\end{gathered}
$$

Proof. Suppose that the conditions (42) and (43) hold, and take any $x=\left(x_{k}\right) \in A_{\lambda}(X)$. Then, the sequence $\left(a_{n k}\right)_{k \in \mathbb{N}} \in$ $\left\{A_{\lambda}(X)\right\}^{\beta}$ for all $n \in \mathbb{N}$, and this implies the existence of the $A$-transform of $x$.
Let us now consider the following equality derived by using the relation (8) from the $m$ th partial sum of the series $\sum_{k} a_{n k} x_{k}$ :

$$
\sum_{k=0}^{m} a_{n k} x_{k}=\sum_{k=0}^{m-1} \tilde{a}_{n k} y_{k}+\frac{\lambda_{m}-\lambda_{m-1}}{\lambda_{m}-2 \lambda_{m-1}+\lambda_{m-2}} a_{n m} y_{m}
$$

for all $m, n \in \mathbb{N}$. Therefore, we obtain from (44) with (42), as $m \rightarrow \infty$, that

$$
\sum_{k} a_{n k} x_{k}=\sum_{k} \widetilde{a}_{n k} y_{k}, \quad \forall n \in \mathbb{N}
$$

Now, by taking the sup-norm in (45), we derive that

$$
\sup _{n \in \mathbb{N}}\left|(A x)_{n}\right| \leq\|y\|_{\infty} \sup _{n \in \mathbb{N}} \sum_{k}\left|\widetilde{a}_{n k}\right|<\infty
$$

which shows the sufficiency of the conditions (42) and (43).

Conversely, suppose that $A=\left(a_{n k}\right) \in\left(A_{\lambda}(X): \ell_{\infty}\right)$. Then, since $\left(a_{n k}\right)_{k \in \mathbb{N}} \in\left\{A_{\lambda}(X)\right\}^{\beta}$ for all $n \in \mathbb{N}$ by the hypothesis, the necessity of (42) is trivial and (45) holds. Consider the continuous linear functionals $f_{n}$ defined on $A_{\lambda}(X)$ by the sequences $a_{n}=\left(a_{n k}\right)_{k \in \mathbb{N}}$ as

$$
f_{n}(x)=\sum_{k} a_{n k} x_{k}, \quad \forall n \in \mathbb{N} .
$$

Since $A_{\lambda}\left(\ell_{\infty}\right) \cong \ell_{\infty}, A_{\lambda}(c) \cong c$ and $A_{\lambda}\left(c_{0}\right) \cong c_{0}$, it should follow with (45) that $\left\|f_{n}\right\|=\left\|\widetilde{a}_{n}\right\|_{\infty}$. This just says that the functionals defined by the rows of $A$ on $A_{\lambda}(X)$ are pointwise bounded. Hence, by Banach-Steinhaus theorem, $f_{n}$ 's are uniformly bounded which gives that there exists a constant $K>0$ such that $\left\|f_{n}\right\| \leq K$ for all $n \in \mathbb{N}$. It therefore follows that $\sum_{k}\left|\tilde{a}_{n k}\right|=\left\|f_{n}\right\| \leq K$ holds for all $n \in \mathbb{N}$ which shows the necessity of the condition (43).

This step completes the proof.

Prior to characterizing the class of infinite matrices from the space $A_{\lambda}\left(\ell_{\infty}\right)$ into the space of almost convergent sequences, we give a short knowledge on the concept of almost convergence. The shift operator $P$ is defined on $\omega$ by $P_{n}(x)=x_{n+1}$ for all $n \in \mathbb{N}$. A Banach limit $L$ is defined on $\ell_{\infty}$, as a non-negative linear functional, such that $L(P x)=L(x)$ and $L(e)=1$. A sequence $x=\left(x_{k}\right) \in \ell_{\infty}$ is said to be almost convergent to the generalized limit $\alpha$ if all Banach limits of $x$ coincide and are equal to $\alpha$ [8] and is denoted by $f-\lim x_{k}=\alpha$. Let $P^{i}$ be the composition of $P$ with itself $i$ times and write for a sequence $x=\left(x_{k}\right)$

$$
t_{m n}(x)=\frac{1}{m+1} \sum_{i=0}^{m} P_{n}^{i}(x), \quad \forall m, n \in \mathbb{N} .
$$

Lorentz [8] proved that $f-\lim x_{k}=\alpha$ if and only if $\lim _{m \rightarrow \infty} t_{m n}(x)=\alpha$, uniformly in $n$. It is well known that a convergent sequence is almost convergent such that its ordinary and generalized limits are equal. By $f_{0}$ and $f$, we denote the spaces of almost null and almost convergent sequences, respectively. Now, we can give the lemma characterizing the almost coercive matrices. 
Lemma 21 (see [9, Theorem 1]). $A=\left(a_{n k}\right) \in\left(\ell_{\infty}: f\right)$ if and only if

$$
\sup _{n \in \mathbb{N}} \sum_{k}\left|a_{n k}\right|<\infty ;
$$

$\exists \alpha_{k} \in \mathbb{C} \ni f-\lim a_{n k}=\alpha_{k} \quad$ for each fixed $k \in \mathbb{N} ;$

$$
\lim _{m \rightarrow \infty} \sum_{k}\left|a(n, k, m)-\alpha_{k}\right|=0 \quad \text { uniformly in } n .
$$

Theorem 22. $A=\left(a_{n k}\right) \in\left(A_{\lambda}\left(\ell_{\infty}\right): f\right)$ if and only if the conditions (42) and (43) hold, and

$$
\begin{gathered}
\exists \alpha_{k} \in \mathbb{C} \ni f-\lim \tilde{a}_{n k}=\tilde{a}_{k} \quad \text { for each fixed } k \in \mathbb{N} ; \\
\lim _{m \rightarrow \infty} \sum_{k}\left|\tilde{a}(n, k, m)-\tilde{a}_{k}\right|=0 \quad \text { uniformly in } n .
\end{gathered}
$$

Proof. Let $A \in\left(A_{\lambda}\left(\ell_{\infty}\right): f\right)$. Then, since $f \subset \ell_{\infty}$, the necessity of (42) and (43) is immediately obtained from Theorem 20 . To prove the necessity of (52), consider the sequence $e^{(n)}(\lambda)=\left\{e_{k}^{(n)}(\lambda)\right\}_{n \in \mathbb{N}} \in A_{\lambda}\left(\ell_{\infty}\right)$, defined by (16) for every fixed $k \in \mathbb{N}$. Since $A x$ exists and is in $f$ for every $x \in A_{\lambda}\left(\ell_{\infty}\right)$, one can easily see that $A e^{(n)}(\lambda)=\left(\widetilde{a}_{n k}\right)_{n \in \mathbb{N}} \in f$ for all $k \in \mathbb{N}$, that is, the condition (52) is necessary.

Define the matrix $B=\left(b_{n k}\right)$ by $b_{n k}=\tilde{a}_{n k}$ for all $k, n \in \mathbb{N}$. Then, we derive from the equality (45) that $A x=B y$. Since $A=\left(a_{n k}\right) \in\left(A_{\lambda}\left(\ell_{\infty}\right): f\right)$ by the hypothesis, we have $B \in$ $\left(\ell_{\infty}: f\right)$. Therefore, the matrix $B$ satisfies the condition (51) of Lemma 21 which is equivalent to the condition (53).

Conversely, suppose that the matrix $A$ satisfies the conditions (42), (43), (52), and (53), and $x \in A_{\lambda}\left(\ell_{\infty}\right)$. Reconsider the equality $A x=B y$ obtained from (45) with $b_{n k}$ instead of $\tilde{a}_{n k}$. Then, the conditions (49), (50), and (51) are satisfied by the matrix $B$. Hence, $B$ is almost coercive by Lemma 21 and this gives by passing to $f$-limit in (45) that $A x \in f$, that is, $A \in\left(A_{\lambda}\left(\ell_{\infty}\right): f\right)$, as desired.

This concludes the proof. lowing.

As a direct consequence of Theorem 22, we have the fol-

Corollary 23. $A=\left(a_{n k}\right) \in\left(A_{\lambda}\left(\ell_{\infty}\right): f_{0}\right)$ if and only if the conditions (42) and (43) hold, and (52) and (53) hold with $\widetilde{a}_{k}=$ 0 for all $k \in \mathbb{N}$.

Theorem 24. $A=\left(a_{n k}\right) \in\left(A_{\lambda}\left(\ell_{\infty}\right): c\right)$ if and only if the condition (42) holds, and the conditions

$$
\sum_{k}\left|\tilde{a}_{n k}\right| \text { converge uniformly in } n \in \mathbb{N} \text {; }
$$

$\exists \alpha_{k} \in \mathbb{C} \ni \lim _{n \rightarrow \infty} a_{n k}=\alpha_{k} \quad$ for each fixed $k \in \mathbb{N}$.

Corollary 25. $A=\left(a_{n k}\right) \in\left(A_{\lambda}\left(\ell_{\infty}\right): c_{0}\right)$ if and only if the conditions (42) and (54) hold, and (55) also holds with $\alpha_{k}=0$ for all $k \in \mathbb{N}$.

Now, we give the following lemma due to King [10] characterizing the class of almost conservative matrices.
Lemma 26. $A=\left(a_{n k}\right) \in(c: f)$ if and only if (49) and (50) hold, and

$$
\exists \alpha \in \mathbb{C} \ni f-\lim \sum_{k} a_{n k}=\alpha .
$$

Theorem 27. $A=\left(a_{n k}\right) \in\left(A_{\lambda}(c): f\right)$ if and only if the conditions (42), (43), and (52) hold, and the condition

$$
\exists \alpha \in \mathbb{C} \ni f-\lim \sum_{k} \widetilde{a}_{n k}=\widetilde{a} .
$$

also holds.

Proof. This is obtained by a similar way used in proving Theorem 22 with Lemma 26 instead of Lemma 21. So, to avoid the repetition of the similar statements we omit the details.

Corollary 28. $A=\left(a_{n k}\right) \in\left(A_{\lambda}(c): f\right)_{\rho}$ if and only if the conditions (42) and (43) hold, and the conditions (52) and (57) also hold with $\alpha_{k}=0$ for all $k \in \mathbb{N}$ and $\widetilde{a}=\rho$, respectively; where by $\left(A_{\lambda}(c): f\right)_{\rho}$, we denote the class of infinite matrices A such that $f-\lim A x=\rho[A(\lambda)-\lim x]$ for all $x \in A_{\lambda}(c)$.

Now, we give the following Steinhaus type theorem.

Theorem 29. The classes $\left(A_{\lambda}\left(\ell_{\infty}\right): f\right)$ and $\left(A_{\lambda}(c): f\right)_{\rho}$ are disjoint, where $\rho \in \mathbb{R} \backslash\{0\}$.

Proof. Suppose that the classes $\left(A_{\lambda}\left(\ell_{\infty}\right): f\right)$ and $\left(A_{\lambda}(c): f\right)_{\rho}$ are not disjoint. Then, there is at least one $A=\left(a_{n k}\right)$ in the set $\left(A_{\lambda}\left(\ell_{\infty}\right): f\right) \cap\left(A_{\lambda}(c): f\right)_{\rho}$. Therefore, one can derive by combining (53) and (52) with $\alpha_{k}=0$ for all $k \in \mathbb{N}$ that

$$
\lim _{m \rightarrow \infty} \sum_{k}|\widetilde{a}(n, k, m)|=0 \quad \text { uniformly in } n
$$

which is contrary to the condition (57) with $\widetilde{a}=\rho \neq 0$. This completes the proof.

Lemma 30 (see [11, Lemma 5.3]). Let $\mu, \nu$ be any two sequence spaces, $A$ an infinite matrix, and $B$ a triangle matrix. Then, $A \in\left(\mu: \nu_{B}\right)$ if and only if $B A \in(\mu: \nu)$.

It is trivial that Lemma 30 has several consequences. Indeed, combining Lemma 30 with Theorems 20, 22, 24, and 27 and Corollaries 23,25 , and 28 by choosing $B$ as one of the special matrices $C_{1}, E^{r}, R^{t}, \Delta, \Delta^{(1)}, A^{r}$, or $S$, one can easily obtain the following results.

Corollary 31. Let $A=\left(a_{n k}\right)$ be an infinite matrix over the complex field. Then, the following statements hold.

(i) $E=\left(e_{n k}\right) \in\left(A_{\lambda}(X): b v_{\infty}\right)$ if and only if (42) and (43) hold with $e_{n k}-e_{n-1, k}$ instead of $a_{n k}$, where $b v_{\infty}$ denotes the space of all sequences $x=\left(x_{k}\right)$ such that $\left(x_{k}-x_{k-1}\right) \in \ell_{\infty}$ and was introduced by Başar and Altay [11].

(ii) $E=\left(e_{n k}\right) \in\left(A_{\lambda}(X): e_{\infty}^{r}\right)$ if and only if (42) and (43) hold with $\sum_{j=0}^{n}\left(\begin{array}{c}n \\ j\end{array}\right)(1-r)^{n-j} r^{j} e_{j k}$ instead of $a_{n k}$, 
where $e_{\infty}^{r}$ denotes the space of all sequences $x=\left(x_{k}\right)$ such that $\left(\sum_{j=0}^{n}\left(\begin{array}{c}n \\ j\end{array}\right)(1-r)^{n-j} r^{j} e_{k j} x_{j}\right) \in \ell_{\infty}$ and was introduced by Altay et al. [12].

(iii) $E=\left(e_{n k}\right) \in\left(A_{\lambda}(X): X_{\infty}\right)$ if and only if (42) and (43) hold with $e(n, k) /(n+1)$ instead of $a_{n k}$, where $X_{\infty}$ denotes the space of all sequences $x=\left(x_{k}\right)$ such that $\left(\sum_{j=0}^{n} x_{j} /(n+1)\right) \in \ell_{\infty}$ and was introduced by $\mathrm{Ng}$ and Lee [13].

(iv) $E=\left(e_{n k}\right) \in\left(A_{\lambda}(X): r_{\infty}^{t}\right)$ if and only if (42) and (43) hold with $\sum_{j=0}^{n} t_{j} e_{j k} / T_{n}$ instead of $a_{n k}$, where $r_{\infty}^{t}$ denotes the space of all sequences $x=\left(x_{k}\right)$ such that $\left(\sum_{j=0}^{n} t_{j} x_{j} / T_{n}\right) \in \ell_{\infty}$ and was introduced by Altay and Başar [14].

(v) $E=\left(e_{n k}\right) \in\left(A_{\lambda}(X): b s\right)$ if and only if (42) and (43) hold with $e(n, k)$ instead of $a_{n k}$.

Corollary 32. Let $A=\left(a_{n k}\right)$ be an infinite matrix over the complex field. Then, the following statements hold.

(i) $E=\left(e_{n k}\right) \in\left(A_{\lambda}\left(\ell_{\infty}\right): c(\Delta)\right)$ if and only if (42), (54), and (55) hold with $e_{n k}-e_{n+1, k}$ instead of $a_{n k}$, where $c(\Delta)$ denotes the space of all sequences $x=\left(x_{k}\right)$ such that $\left(x_{k}-x_{k+1}\right) \in c$ and was introduced by Kizmaz [15].

(ii) $E=\left(e_{n k}\right) \in\left(A_{\lambda}\left(\ell_{\infty}\right): e_{c}^{r}\right)$ if and only if (42), (54), and (55) hold with $\sum_{j=0}^{n}\left(\begin{array}{c}n \\ j\end{array}\right)(1-r)^{n-j} r^{j} e_{j k}$ instead of $a_{n k}$, where $e_{c}^{r}$ denotes the space of all sequences $x=\left(x_{k}\right)$ such that $E^{r} x \in c$ and was introduced by Altay and Başar [16].

(iii) $E=\left(e_{n k}\right) \in\left(A_{\lambda}\left(\ell_{\infty}\right): \widetilde{c}\right)$ if and only if (42), (54), and (55) hold with $e(n, k) /(n+1)$ instead of $a_{n k}$, where $\tilde{c}$ denotes the space of all sequences $x=\left(x_{k}\right)$ such that $C_{1} x \in c$ and was introduced by Şengönül and Başar [17].

(iv) $E=\left(e_{n k}\right) \in\left(A_{\lambda}\left(\ell_{\infty}\right): r_{c}^{t}\right)$ if and only if (42), (54), and (55) hold with $\sum_{j=0}^{n} t_{j} e_{j k} / T_{n}$ instead of $a_{n k}$, where $r_{c}^{t}$ denotes the space of all sequences $x=\left(x_{k}\right)$ such that $R^{t} x \in c$ and was introduced by Altay and Başar [18].

(v) $E=\left(e_{n k}\right) \in\left(A_{\lambda}\left(\ell_{\infty}\right): c s\right)$ if and only if (42), (54) and (55) hold with $e(n, k)$ instead of $a_{n k}$.

Corollary 33. Let $A=\left(a_{n k}\right)$ be an infinite matrix over the complex field. Then, the following statements hold.

(i) $A=\left(a_{n k}\right) \in\left(A_{\lambda}\left(\ell_{\infty}\right): \widehat{f}\right)$ if and only if (42), (43), (52), and (53) hold with $d_{n k}$ instead of $a_{n k}$, where $\hat{f}$ denotes the space of all sequences $x=\left(x_{k}\right)$ such that $B(r, s) x \in f$ and was introduced by Başar and Kiriş̧̧ [19].

(ii) $A=\left(a_{n k}\right) \in\left(A_{\lambda}\left(\ell_{\infty}\right): \widehat{f}_{0}\right)$ if and only if (42) and (43) hold, and (52) and (53) hold with $\alpha_{k}=0$ for all $k \in \mathbb{N}$ and $d_{n k}$ instead of $a_{n k}$, where $\widehat{f}_{0}$ denotes the space of all sequences $x=\left(x_{k}\right)$ such that $B(r, s) x \in f_{0}$ and was introduced by Başar and Kiriş̧̧i [19]. (iii) $A=\left(a_{n k}\right) \in\left(A_{\lambda}(c): \widehat{f}\right)$ if and only if (42), (43), (52), and (53) hold with $d_{n k}$ instead of $a_{n k}$.

(iv) $A=\left(a_{n k}\right) \in\left(A_{\lambda}(c): \widehat{f}_{0}\right)$ if and only if (42) and (43) hold, and (52) and (53) also hold with $\alpha_{k}=0$ for all $k \in \mathbb{N}$ and $\alpha=1$, respectively, hold with $\alpha_{k}=0$ for all $k \in \mathbb{N}$ and $d_{n k}$ instead of $a_{n k}$.

(v) $A=\left(a_{n k}\right) \in\left(A_{\lambda}(c): \widehat{f}\right)_{\rho}$ if and only if the conditions of Corollary 28 hold with $d_{n k}$ instead of $a_{n k}$.

Corollary 34. Let $A=\left(a_{n k}\right)$ be an infinite matrix over the complex field. Then, the following statements hold.

(i) $A=\left(a_{n k}\right) \in\left(A_{\lambda}\left(\ell_{\infty}\right): \tilde{f}\right)$ if and only if (42), (43), (52), and (53) hold with $c_{n k}$ instead of $a_{n k}$, where $\tilde{f}$ denotes the space of all sequences $x=\left(x_{k}\right)$ such that $C_{1} x \in f$ and was introduced by Kayaduman and Şengönül [20].

(ii) $A=\left(a_{n k}\right) \in\left(A_{\lambda}\left(\ell_{\infty}\right): \tilde{f}_{0}\right)$ if and only if (42) and (43) hold, and (52) and (53) hold with $\alpha_{k}=0$ for all $k \in \mathbb{N}$ and $c_{n k}$ instead of $a_{n k}$, where $\tilde{f}_{0}$ denotes the space of all sequences $x=\left(x_{k}\right)$ such that $C_{1} x \in f_{0}$ and was introduced by Kayaduman and Şengönül [20].

(iii) $A=\left(a_{n k}\right) \in\left(A_{\lambda}(c): \tilde{f}\right)$ if and only if (42), (43), (52), and (53) hold with $c_{n k}$ instead of $a_{n k}$.

(iv) $A=\left(a_{n k}\right) \in\left(A_{\lambda}(c): \widetilde{f}_{0}\right)$ if and only if (42) and (43) hold, and (52) and (53) also hold with $\alpha_{k}=0$ for all $k \in \mathbb{N}$ and $\alpha=1$, respectively, with $c_{n k}$ instead of $a_{n k}$.

(v) $A=\left(a_{n k}\right) \in\left(A_{\lambda}(c): \widetilde{f}\right)_{\rho}$ if and only if the conditions of Corollary 28 hold with $c_{n k}$ instead of $a_{n k}$.

Corollary 35. Let $A=\left(a_{n k}\right)$ be an infinite matrix over the complex field. Then, the following statements hold.

(i) $A=\left(a_{n k}\right) \in\left(A_{\lambda}\left(\ell_{\infty}\right): f(B)\right)$ if and only if (42), (43), (52), and (53) hold with $e_{n k}$ instead of $a_{n k}$, where $f(B)$ denotes the space of all sequences $x=\left(x_{k}\right)$ such that $B(r, s, t) x \in f$ and was introduced by Sönmez [21].

(ii) $A=\left(a_{n k}\right) \in\left(A_{\lambda}\left(\ell_{\infty}\right): f_{0}(B)\right)$ if and only if (42) and (43) hold, and (52) and (53) hold with $\alpha_{k}=0$ for all $k \in \mathbb{N}$ and $e_{n k}$ instead of $a_{n k}$, where $f_{0}(B)$ denotes the space of all sequences $x=\left(x_{k}\right)$ such that $B(r, s, t) x \in f_{0}$ and was introduced by Sönmez [21].

(iii) $A=\left(a_{n k}\right) \in\left(A_{\lambda}(c): f(B)\right)$ if and only if the conditions (42), (43), (52), and (53) hold with $e_{n k}$ instead of $a_{n k}$.

(iv) $A=\left(a_{n k}\right) \in\left(A_{\lambda}(c): f_{0}(B)\right)$ if and only if the conditions (42) and (43) hold, and the conditions (52) and (53) also hold with $\alpha_{k}=0$ for all $k \in \mathbb{N}$ and $\alpha=1$, respectively, hold with $\alpha_{k}=0$ for all $k \in \mathbb{N}$ and $e_{n k}$ instead of $a_{n k}$.

(v) $A=\left(a_{n k}\right) \in\left(A_{\lambda}(c): f(B)\right)_{\rho}$ if and only if the conditions of Corollary 28 hold with $e_{n k}$ instead of $a_{n k}$.

\section{Conclusion}

Mursaleen and Noman [2, 22, 23] have studied the domains $\ell_{\infty}^{\lambda}, c^{\lambda}, c_{0}^{\lambda}$, and $\ell_{p}^{\lambda}$ of the matrix $\Lambda$ in the classical 
sequence spaces $\ell_{\infty}, c, c_{0}$, and $\ell_{p}$, respectively. Malkowsky and Rakočević [24] characterized some classes of matrix transformations and investigated related compact operators involving the spaces of $\Lambda$-null, $\Lambda$-convergent, and $\Lambda$-bounded sequences. Quite recently, Sönmez and Başar [25] have introduced the spaces $c_{0}^{\lambda}(B)$ and $c^{\lambda}(B)$ of generalized difference sequences which generalize the paper due to Mursaleen and Noman [26]. Mursaleen and Noman [26] have derived some inclusion relations and determined the alpha-, beta-, and gamma-duals of those spaces and constructed their Schauder bases. Finally, Sönmez and Başar [25] have characterized some matrix classes from the spaces $c_{0}^{\lambda}(B)$ and $c^{\lambda}(B)$ to the spaces $\ell_{p}, c_{0}$, and $c$. In the present paper, we emphasize the domains $A_{\lambda}\left(c_{0}\right), A_{\lambda}(c)$, and $A_{\lambda}\left(\ell_{\infty}\right)$ of the matrix $A(\lambda)$ in the classical sequence spaces $c_{0}, c$, and $\ell_{\infty}$. Our results are more general and comprehensive than the corresponding results of Mursaleen and Noman $[2,22,23]$ derived with the matrix $\Lambda$. We should note that, as a natural continuation of the present paper, one can study the domains $A_{\lambda}\left(\ell_{p}\right)$ and $A_{\lambda}\left(b v_{p}\right)$ of the matrix $A(\lambda)$ in the classical sequence space $\ell_{p}$ and in the sequence space $b v_{p}$ with $0<p<1$ and $1 \leq p<\infty$, where $b v_{p}$ denotes the space of all sequences $x=\left(x_{k}\right)$ such that $\left(x_{k}-x_{k-1}\right) \in \ell_{p}$ and introduced in the case $1 \leq p<\infty$ by Başar and Altay [11] and in the case $0<p<1$ by Altay and Başar [27].

\section{Acknowledgment}

The authors would like to express their pleasure to the anonymous referees for constructive criticism of an earlier version of this paper which improved its readability.

\section{References}

[1] N. L. Braha, "A new class of sequences related to the $l_{p}$ spaces defined by sequences of Orlicz functions," Journal of Inequalities and Applications, vol. 2011, Article ID 539745, 10 pages, 2011.

[2] M. Mursaleen and A. K. Noman, "On the spaces of $\lambda$-convergent and bounded sequences," Thai Journal of Mathematics, vol. 8, no. 2, pp. 311-329, 2010.

[3] M. Stieglitz and H. Tietz, "Matrixtransformationen von folgenräumen. Eine ergebnisübersicht," Mathematische Zeitschrift, vol. 154, no. 1, pp. 1-16, 1977.

[4] E. Malkowsky and E. Savaş, "Matrix transformations between sequence spaces of generalized weighted means," Applied Mathematics and Computation, vol. 147, no. 2, pp. 333-345, 2004.

[5] N. L. Braha and T. Mansour, "On $\Lambda^{2}$-strong convergence of numerical sequences and Fourier series," Acta Mathematica Hungarica, 2013.

[6] A. Wilansky, Summability Through Functional Analysis, vol. 85 of North-Holland Mathematics Studies, Elsevier, New York, NY, USA, 1984.

[7] F. Başar, Summability Theory and Its Applications, Monographs, Bentham Science Publishers E-books, İstanbul, Turkey, 2012.

[8] G. G. Lorentz, "A contribution to the theory of divergent sequences," Acta Mathematica, vol. 80, pp. 167-190, 1948.

[9] J. P. Duran, "Infinite matrices and almost-convergence," Mathematische Zeitschrift, vol. 128, pp. 75-83, 1972.
[10] J. P. King, "Almost summable sequences," Proceedings of the American Mathematical Society, vol. 17, pp. 1219-1225, 1966.

[11] F. Başar and B. Altay, "On the space of sequences of $p$ bounded variation and related matrix mappings," Ukrainian Mathematical Journal, vol. 55, no. 1, pp. 136-147, 2003.

[12] B. Altay, F. Başar, and M. Mursaleen, "On the Euler sequence spaces which include the spaces $\ell_{p}$ and $\ell_{\infty} I$," Information Sciences, vol. 176, no. 10, pp. 1450-1462, 2006.

[13] P. N. Ng and P. Y. Lee, "Cesàro sequence spaces of non-absolute type," Commentationes Mathematicae. Prace Matematyczne, vol. 20, no. 2, pp. 429-433, 1978.

[14] B. Altay and F. Başar, "On the paranormed Riesz sequence spaces of non-absolute type," Southeast Asian Bulletin of Mathematics, vol. 26, no. 5, pp. 701-715, 2003.

[15] H. Kizmaz, "On certain sequence spaces," Canadian Mathematical Bulletin, vol. 24, no. 2, pp. 169-176, 1981.

[16] B. Altay and F. Başar, "Some Euler sequence spaces of nonabsolute type," Ukrainian Mathematical Journal, vol. 57, no. 1, pp. $1-17,2005$.

[17] M. Şengönül and F. Başar, "Some new Cesàro sequence spaces of non-absolute type which include the spaces $\mathcal{c}_{0}$ and $c$," Soochow Journal of Mathematics, vol. 31, no. 1, pp. 107-119, 2005.

[18] B. Altay and F. Başar, "Some paranormed Riesz sequence spaces of non-absolute type," Southeast Asian Bulletin of Mathematics, vol. 30, no. 4, pp. 591-608, 2006.

[19] F. Başar and M. Kirișçi, "Almost convergence and generalized difference matrix," Computers \& Mathematics with Applications, vol. 61, no. 3, pp. 602-611, 2011.

[20] K. Kayaduman and K. Şengönül, “The spaces of Cesàro almost convergent sequences and core theorems," Acta Mathematica Scientia B, vol. 32, no. 6, pp. 2265-2278, 2012.

[21] A. Sönmez, "Almost convergence and triple band matrix," Mathematical and Computer Modelling, vol. 57, no. 9-10, pp. 2393-2402, 2013.

[22] M. Mursaleen and A. K. Noman, "On some new sequence spaces of non-absolute type related to the spaces $\ell_{p}$ and $\ell_{\infty} I$," Filomat, vol. 25, no. 2, pp. 33-51, 2011.

[23] M. Mursaleen and A. K. Noman, "On some new sequence spaces of non-absolute type related to the spaces $\ell_{p}$ and $\ell_{\infty} I I$," Mathematical Communications, vol. 16, no. 2, pp. 383-398, 2011.

[24] E. Malkowsky and V. Rakočević, "Measure of noncompactness of linear operators between spaces of sequences that are $(\bar{N}, q)$ summable or bounded," Czechoslovak Mathematical Journal, vol. 51, no. 3, pp. 505-522, 2001.

[25] A. Sönmez and F. Başar, "Generalized difference spaces of nonabsolute type of convergent and null sequences," Abstract and Applied Analysis, vol. 2012, Article ID 435076, 20 pages, 2012.

[26] M. Mursaleen and A. K. Noman, "On some new difference sequence spaces of non-absolute type," Mathematical and Computer Modelling, vol. 52, no. 3-4, pp. 603-617, 2010.

[27] B. Altay and F. Başar, "The fine spectrum and the matrix domain of the difference operator $\Delta$ on the sequence space $\ell_{p}, 0<p<$ 1," Communications in Mathematical Analysis, vol. 2, no. 2, pp. $1-11,2007$. 


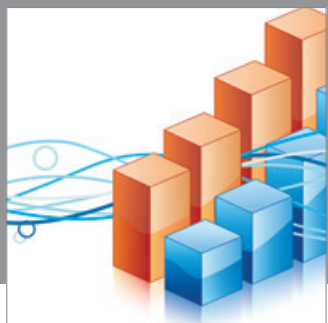

Advances in

Operations Research

mansans

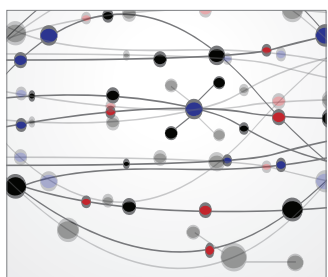

The Scientific World Journal
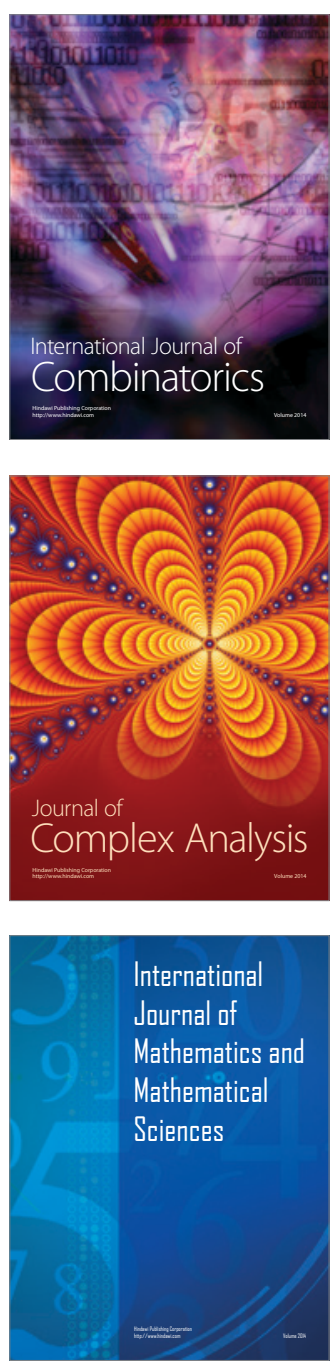
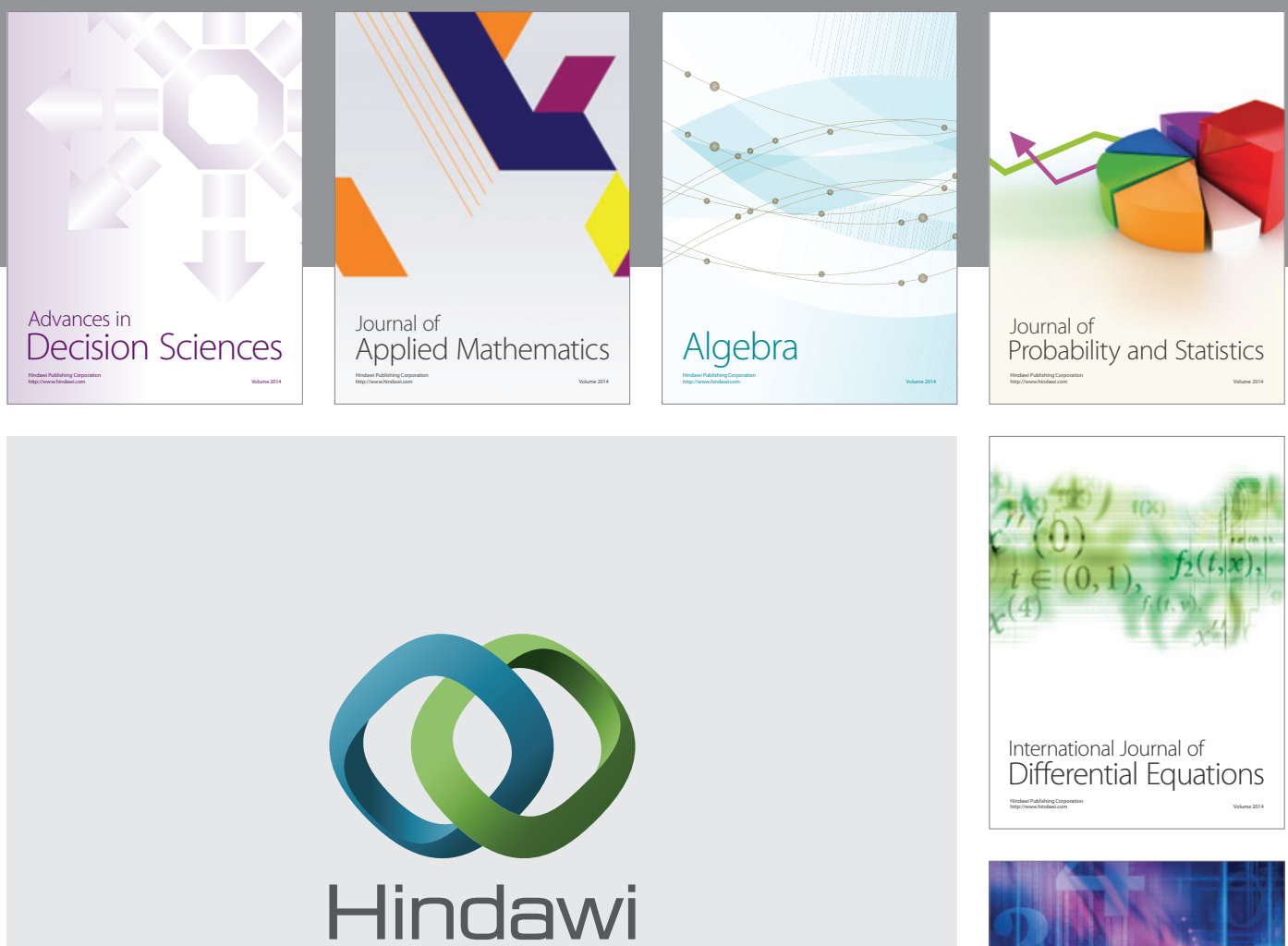

Submit your manuscripts at http://www.hindawi.com
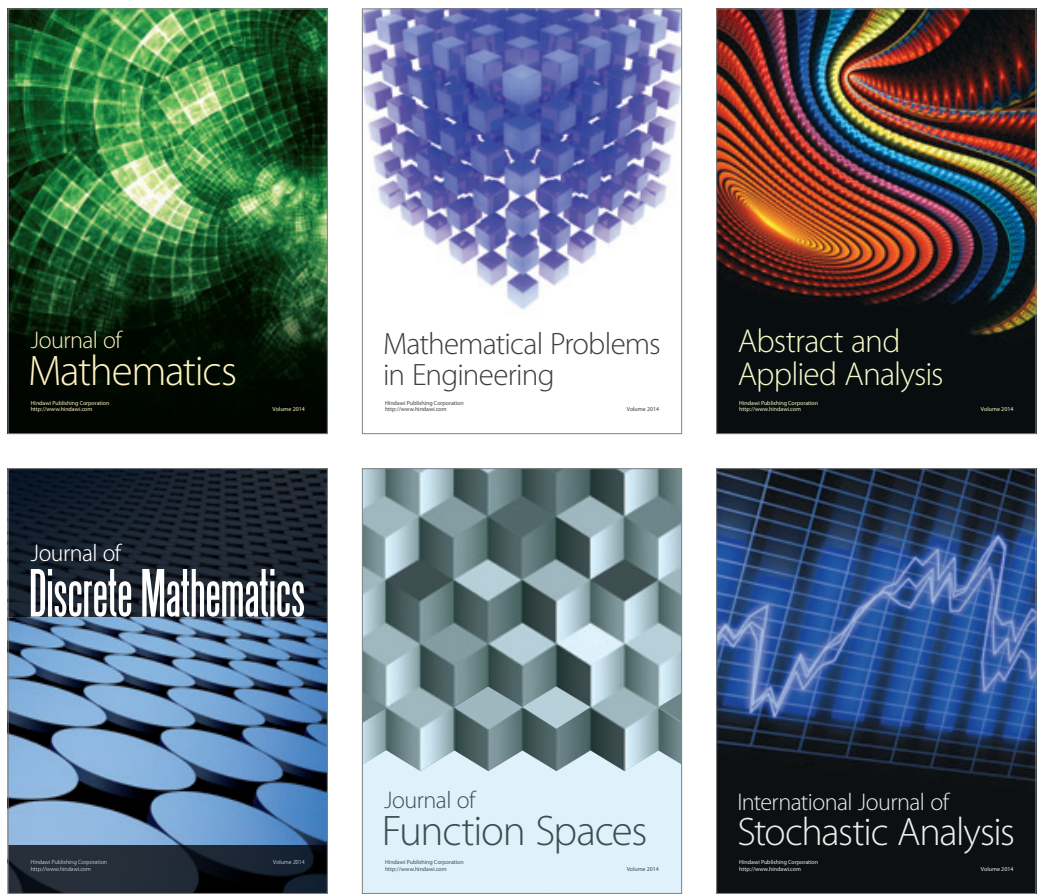

Journal of

Function Spaces

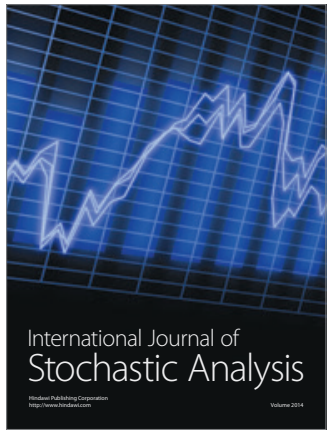

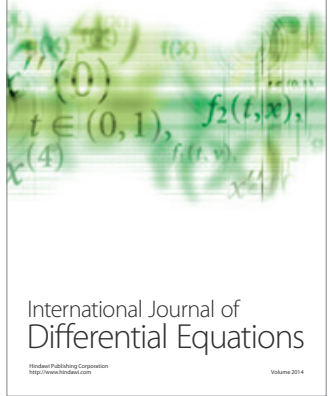
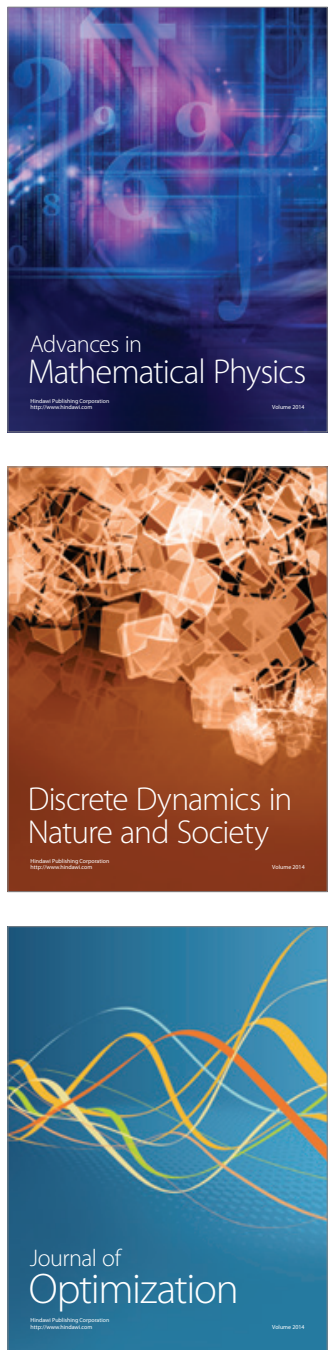